\title{
Development of a cooperative two-factor adaptive-evolution method to enhance lipid production and prevent lipid peroxidation in Schizochytrium sp.
}

Xiao-Man Sun ${ }^{1}$, Lu-Jing Ren ${ }^{1,4^{*}}$, Zhi-Qian Bi ${ }^{1}$, Xiao-Jun Ji ${ }^{1,4}$, Quan-Yu Zhao ${ }^{2}$, Ling Jiang ${ }^{4}$ and He Huang $2,3,4^{*}$

\begin{abstract}
Background: Schizochytrium sp. is a marine microalga with great potential as a promising sustainable source of lipids rich in docosahexaenoic acid (DHA). This organism's lipid accumulation machinery can be induced by various stress conditions, but this stress induction usually comes at the expense of lower biomass in industrial fermentations. Moreover, oxidative damage induced by various environmental stresses can result in the peroxidation of lipids, and especially polyunsaturated fatty acids, which causes unstable DHA production, but is often ignored in fermentation processes. Therefore, it is urgent to develop new production strains that not only have a high DHA production capacity, but also possess strong antioxidant defenses.
\end{abstract}

Results: Adaptive laboratory evolution (ALE) is an effective method for the development of beneficial phenotypes in industrial microorganisms. Here, a novel cooperative two-factor ALE strategy based on concomitant low temperature and high salinity was applied to improve the production capacity of Schizochytrium sp. Low-temperature conditions were used to improve the DHA content, and high salinity was applied to stimulate lipid accumulation and enhance the antioxidative defense systems of Schizochytrium sp. After 30 adaptation cycles, a maximal cell dry weight of $126.4 \mathrm{~g} / \mathrm{L}$ and DHA yield of $38.12 \mathrm{~g} / \mathrm{L}$ were obtained in the endpoint strain ALE-TF30, which was 27.42 and $57.52 \%$ higher than parental strain, respectively. Moreover, the fact that ALE-TF30 had the lowest concentrations of reactive oxygen species and malondialdehyde among all strains indicated that lipid peroxidation was greatly suppressed by the evolutionary process. Accordingly, the ALE-TF30 strain exhibited an overall increase of gene expression levels of antioxidant enzymes and polyketide synthases compared to the parental strain.

Conclusion: This study provides important clues on how to overcome the negative effects of lipid peroxidation on DHA production in Schizochytrium sp. Taken together, the cooperative two-factor ALE process can not only increase the accumulation of lipids rich in DHA, but also prevent the loss of produced lipid caused by lipid peroxidation. The strategy proposed here may provide a new and alternative direction for the industrial cultivation of oil-producing microalgae.

Keywords: Schizochytrium sp., Docosahexaenoic acid, Adaptive evolution, Lipid peroxidation, Antioxidant enzyme

\footnotetext{
*Correspondence: renlujing@njtech.edu.cn; biotech@njtech.edu.cn

${ }^{1}$ College of Biotechnology and Pharmaceutical Engineering, Nanjing

Tech University, No. 30 South Puzhu Road, Nanjing 211816, People's

Republic of China

${ }^{2}$ School of Pharmaceutical Sciences, Nanjing Tech University, No. 30

South Puzhu Road, Nanjing 211816, People's Republic of China

Full list of author information is available at the end of the article
} 


\section{Background}

Docosahexaenoic acid (DHA) plays important roles in alleviating cardiovascular diseases, hypertension, diabetes, and neuro-psychiatric disorders [1]. Due to these positive effects on human health, DHA is of considerable interest as a food additive and pharmaceutical target. Marine fish are traditionally known as a natural source of DHA. However, the limitations of this source, such as seasonal availability, location, the ecological impact of fishing, and the resulting environmental pollution, make it difficult to fulfill the nutritional requirements of human beings [2]. In fact, the accumulation of DHA in marine fish is largely attributed to their consumption of microalgae. Hence, microalgae have a significant potential as sources of omega-3 polyunsaturated fatty acids (PUFA). The marine microalga Schizochytrium sp. is famous for producing significant amounts of DHA, in conjunction with a fast growth rate and high productivity [3].

In general, lipids act as an energy-rich carbon storage battery, and are synthesized by microalgae to retain their growth rate under extreme environmental conditions such as nitrogen limitation or high salinity [4]. Consequently, stress-based strategies are widely used as environmentally friendly approaches to induce lipid overproduction in cultured microorganisms [5]. However, stress-based strategies usually work at the expense of lower biomass. A general countermeasure to overcome this difficulty is to use a two-stage cultivation strategy, dedicating the first stage with optimum growth conditions to the maximization of biomass production, while reserving the second stage for the accumulation of lipids under stress conditions. However, such multi-stage cultivation strategies consume more energy, and the exact time-point of switching the conditions for fermentation control is difficult to determine accurately, leading to high costs and production instability [6]. Consequently, stable mutants with improved tolerance to the process conditions should be developed to enhance lipid yield.

Moreover, formation of reactive oxygen species (ROS) is an inevitable aspect of cellular responses to stressbased strategies. It is well established that ROS can react instantaneously and non-specifically with essential biological molecules, and lead to the alteration of cellular functions by inducing DNA damage and mutations, protein oxidation, or peroxidation of lipids [7]. The lipids of microalgae contain a high percentage of PUFAs and are consequently susceptible to peroxidation because the unsaturated double bonds in the PUFAs are more prone to oxidation [8]. Lipid peroxidation not only leads to the accumulation of high levels of ROS, but also causes a loss of the produced lipids, which unfortunately must often be ignored in fermentation processes. Cells experience oxidative stress when the amount of ROS exceeds the capacity of the antioxidant defense systems to detoxify them [9]. Hence, it is urgent to obtain a strain of Schizochytrium sp. with combined characteristics of high production and strong antioxidant defense systems to prevent lipid peroxidation. There are several approaches available to achieve this end, mostly involving genetic engineering techniques. However, the use of genetically modified strains in the food industry is not permitted in many parts of the world, and consumer acceptance of such technologies remains a contentious issue [10]. To overcome these bottlenecks, an approach such as adaptive laboratory evolution (ALE) could be beneficial.

ALE has been widely used to develop novel biological and phenotypic functions, and also for the improvement of strains obtained through synthetic biology, including important model organisms such as Saccharomyces cerevisiae and Escherichia coli [11]. Briefly, ALE subjects microbes to repeated or continuous cultivation under stress conditions for many generations to enrich favorable genetic changes or achieve better inhibitor tolerance [12]. In recent years, many successful ALE experiments were applied to improve the production of lipids and other high-value-added products in microalgae. For example, a highly glucose-tolerant strain of Crypthecodinium cohnii and a highly glycerol-tolerant strain of Rhodococcus opacus were obtained by ALE to relieve substrate inhibition, which exhibited improved growth and lipid accumulation at high glucose and glycerol concentrations, respectively $[13,14]$. In addition to nutrient stress, various environmental stress factors such as high oxygen [15], high temperature [16], or high salt concentrations [17] were applied in ALE to improve microalgal performance. Thus, ALE has been gradually introduced as a novel, powerful tool to improve the strain properties of microalgae. The increase of the PUFA percentage at low culture temperatures is a common trend reported for microalgae. For example, a temperature decrease yielded two- to threefold increase of EPA and DHA contents in several mesophilic species $[18,19]$. In addition, many recent studies have found significant effects of salinity on the concentrations of important antioxidants in microalgae. In Dunaliella tertiolecta, hypersaline stress can induce a massive increase of the hydrogen peroxide scavenging capacity [20]. We therefore hypothesized that a combined low-temperature/high-salinity adaption strategy could be applied to simultaneously improve DHA synthesis and antioxidant defense systems of Schizochytrium sp., which would arguably lead to better industrial strains.

Very few studies to date have focused on the effects of oxidative damage on the fermentation performance of microalgae. In addition, we are not aware of any studies devoted to the development of a high antioxidant 
capacity in this organism. In this study, we successfully established a cooperative two-factor ALE strategy, including low temperature and high salinity, to simultaneously improve the growth and DHA production of Schizochytrium sp. The best evolved clones exhibited significantly increased product formation and high antioxidant capacity. ROS, T-AOC, and MDA were used to compare the antioxidant defense systems of the endpoint strain and starting strain. In addition, seven genes associated with antioxidant enzymes and fatty acid biosynthesis were analyzed by qRT-PCR. This study thus provides a new ALE method that can improve multiple factors of strain productivity, which also might be used in other oilproducing microalgae.

\section{Results}

\section{Changes of the growth properties of Schizochytrium sp. during ALE}

We analyzed samples collected each 2 cycles to monitor the cell dry weight (CDW) and analyzed the fatty acid profiles each 10 cycles. These two indices were recognized as the first indicators of the success or failure of the adaption. As shown in Fig. 1a, at the end of the second cycle, the biomass concentration under low temperature and high salinity reached 15.0 and $12.1 \mathrm{~g} / \mathrm{L}$, which was 53.7 and $62.7 \%$ lower than that of the control, respectively. Furthermore, the combination of the two stress conditions resulted in a severe drop of $\mathrm{CDW}$ to $10.4 \mathrm{~g} / \mathrm{L}$, indicating that combined stresses hinder the growth of Schizochytrium sp. more than the individual stress factors. With the progress of the ALE process, a clear increasing trend of biomass became visible in spite of considerable fluctuations. The strains undergoing lowtemperature ALE took 18 cycles to recover the original growth rate under optimal conditions, at which point the CDW was $32.4 \mathrm{~g} / \mathrm{L}$ - the same as that of the control. By contrast, the recovery of the original CDW took 22 cycles in the high-salinity ALE, indicating that Schizochytrium sp. requires a greater length of time to obtain a stable improved phenotype under high-salinity stress. Similarly, strains in the cooperative two-factor ALE took the longest to adapt, reaching the CDW of the control only after 28 cycles. In spite of the difficulty of adaptation, ALELT30, ALE-HS30, and ALE-TF30 all showed increased growth compared to the parental strain.

Moreover, a significant difference was observed in the fatty acid composition between the staring strain and the evolved strains (Fig. 1). The saturated fatty acids (SFA) in Schizochytrium sp. are mainly composed of C14:0 and C16:0, whereas PUFAs mainly consist of EPA, docosapentaenoic acid (DPA), and DHA. As shown in Fig. 1, the strains' biosynthesis capacity for SFA and PUFA followed opposite trajectories under all adaptation conditions.
With increasing adaptation time under low temperature, the PUFA percentage in TFAs increased over time from $67.11 \%$ at the beginning to $80.22 \%$ at 30 cycles. Importantly, EPA accumulation increased significantly after 20 cycles of low-temperature ALE, resulting in an EPA content of $6.23 \%$ of TFAs, which was 4.6 -fold higher than that of the starting strain. Moreover, the DHA percentage showed a peak value $55.70 \%$ after 30 cycles, which was $12.91 \%$ higher than that of the starting strain. However, an opposite trend was observed in the high-salinity ALE, whereby SFA increased and PUFA decreased over time. Unfortunately, the PUFA percentage continued to decrease and finally reached $54.59 \%$ in the endpoint strain ALE-HS30, which was $18.65 \%$ lower than that of the starting strain. Cooperative two-factor ALE also enhanced the PUFA percentage during the entire time course, although the increment was lower than in the low-temperature ALE experiment, reflecting the strong influence of low-temperature stress on fatty acid composition.

\section{Isolation and comparison of the most promising evolved strains}

In an attempt to evaluate the effects of the three ALE methods and isolate the most promising evolved strains, we investigated the changes of the fermentation characteristics between the starting strain and the endpoint strains ALE-LT30, ALE-HS30, and ALE-TF30, cultured under the same conditions at $30{ }^{\circ} \mathrm{C}$ in normal fermentation medium. All of the strains were able to complete the fermentation, although the duration of the fermentation differed between the evolved strains (Table 1). Two evolved strains, ALE-SH30 and ALE-TF30, consumed all of the sugar in less than $45 \mathrm{~h}$, the same time as the ancestral strain, whereas ALE-LT30 took an additional $8 \mathrm{~h}$. Finally, the observation that the CDW of ALE-LT30 was the lowest further reflected the fact that the lowtemperature adaptation actually hindered the cell growth of Schizochytrium sp. By contrast, the biomass concentrations of the evolved strains ALE-HS30 and ALE-TF30 were increased to 49.85 and $47.23 \mathrm{~g} / \mathrm{L}$, which were 23.39 and $16.91 \%$ higher than in the parental strain, respectively (Table 1 ). Moreover, the strains adapted to high salinity, including ALE-HS30 and ALE-TF30, exhibited positive changes of lipid accumulation. In these cultures, the maximum lipid production achieved by ALE-HS30 was $20.45 \mathrm{~g} / \mathrm{L}$, representing $41.02 \%$ of CDW after $42-\mathrm{h}$ cultivation, which was $51.48 \%$ higher than that of the parental strain.

Interestingly, ALE exhibited significant effects not only on the total lipids, but also on the fatty acid composition (Fig. 2). Similarly, the PUFA percentage of the ALELT30 strain increased to $79.62 \%$ and the SFA percentage 

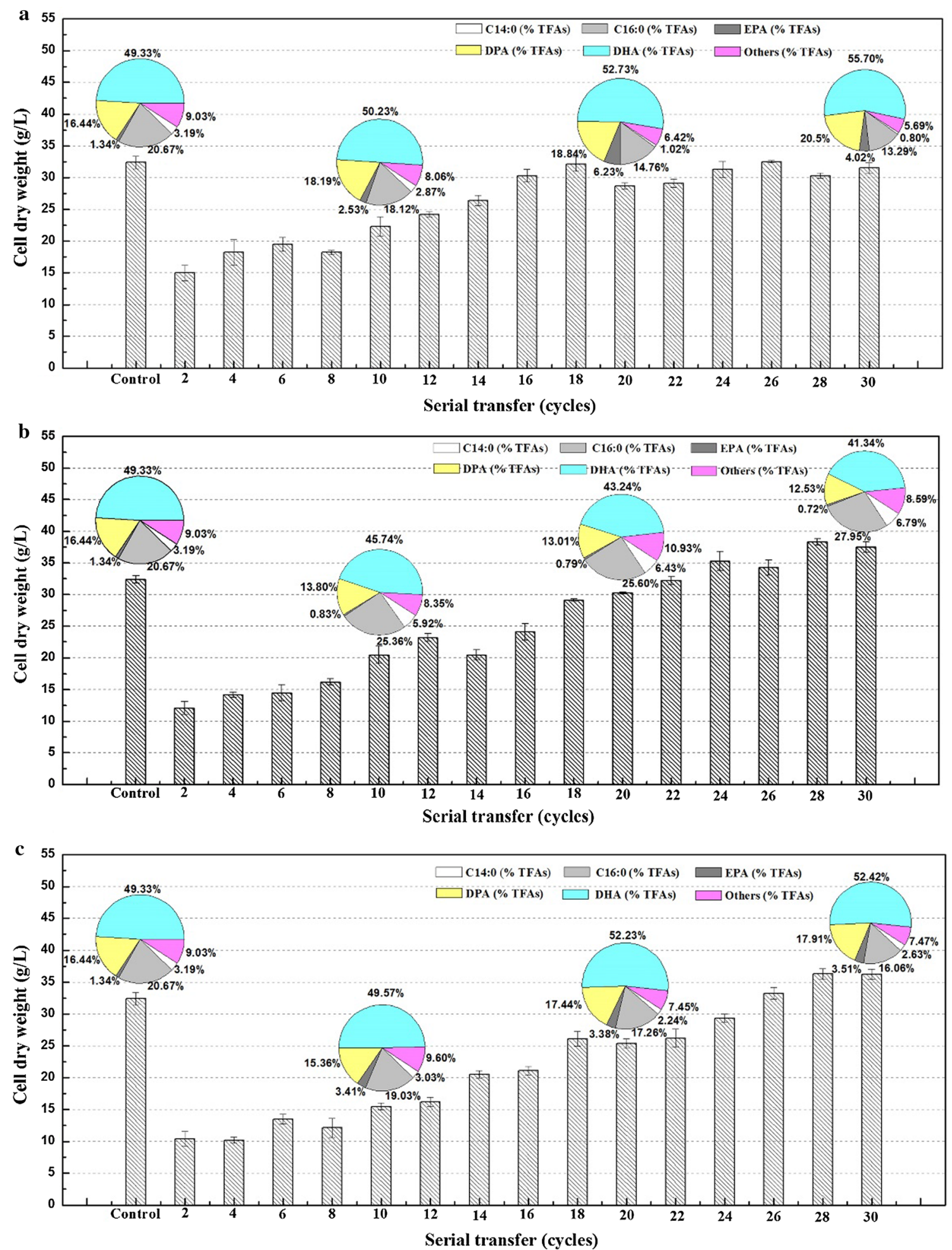

Fig. 1 Cell dry weight (CDW) and fatty acid content (as \% of TFAs) of different evolved strains during adaptive laboratory evolution. Values and error bars represent the means and the standard deviations of triplicate experiments. a Low-temperature ALE, $\mathbf{b}$ high-salinity ALE, $\mathbf{c}$ cooperative two-factor ALE 
Table 1 Fermentation characteristics of different evolved strains at the end of fermentation

\begin{tabular}{|c|c|c|c|c|}
\hline \multirow[t]{2}{*}{ Parameter } & \multicolumn{4}{|l|}{ Strain } \\
\hline & Parental strain & ALE-LT30 & ALE-HS30 & ALE-TF30 \\
\hline CDW (g/L) & $40.4 \pm 0.8$ & $36.10 \pm 1.1$ & $49.85 \pm 1.3$ & $47.23 \pm 0.9$ \\
\hline $\begin{array}{l}\text { TL concentration } \\
(\mathrm{g} / \mathrm{L})\end{array}$ & $13.5 \pm 0.6$ & $10.13 \pm 0.8$ & $20.45 \pm 0.5$ & $17.76 \pm 1.2$ \\
\hline $\begin{array}{l}\text { TLs in CDW } \\
(w / w, \%)\end{array}$ & 33.41 & 28.06 & 41.02 & 37.60 \\
\hline $\begin{array}{l}\text { Fermentation } \\
\text { time (h) }\end{array}$ & 42 & 50 & 42 & 45 \\
\hline DHA yield (g/L) & $6.58 \pm 0.4$ & $5.49 \pm 0.5$ & $9.04 \pm 0.8$ & $9.49 \pm 0.5$ \\
\hline PUFA/SFA & 2.96 & 5.20 & 2.06 & 4.56 \\
\hline
\end{tabular}

Data represent the mean values and standard deviations of three replicates for each measurement

decreased to $15.32 \%$, compared with 68.82 and $23.23 \%$ in the staring strain, respectively. Thus, the low-temperature ALE strains still kept a significant advantage in EPA accumulation. Conversely, a higher SFA percentage $(28.68 \%)$ and lower PUFA percentage $(59.15 \%)$ were observed with the evolved strain ALE-HS30, which indicated that high-salinity adaptation was advantageous for improving cell growth and lipid production, albeit at the expense of the PUFA yield. Interestingly, ALE-TF30 combined the advantages of higher PUFA accumulation of the low-temperature ALE and higher cell growth and lipid accumulation of the high-salinity ALE. At the end of fermentation, the lipid production and DHA percentage of ALE-TF30 reached $17.76 \mathrm{~g} / \mathrm{L}$ and $53.43 \%$, respectively, thus giving a maximum DHA yield of $9.49 \mathrm{~g} / \mathrm{L}$, which was $44.22 \%$ higher than that of the parental strain. The ALE-TF30 strain therefore was the most promising evolved strain in terms of fermentation performance and DHA production. Moreover, the results of a passage experiment showed that endpoint strain ALE-TF30 had stable genetics in terms of producing a higher cell dry weight and DHA percentage in TFAs (Additional file 1: Table S1).

To further explore the stress status of the parental strain and the endpoint strain under the tested experimental conditions, the morphology and oxidative stress of the cells were investigated by confocal laser microscopy. Intracellular ROS react with the DCFH-DA probe to produce an oxidized fluorophore exhibiting green fluorescence at an excitation wavelength of $485 \mathrm{~nm}$ with an emission wavelength of $530 \mathrm{~nm}$, whereby the fluorescence intensity reflects the degree of oxidative damage in the cells. Figure 3B shows the data from the parental strain cultured under combined low-temperature/highsalinity conditions for 1 cycle. At this point, the cells
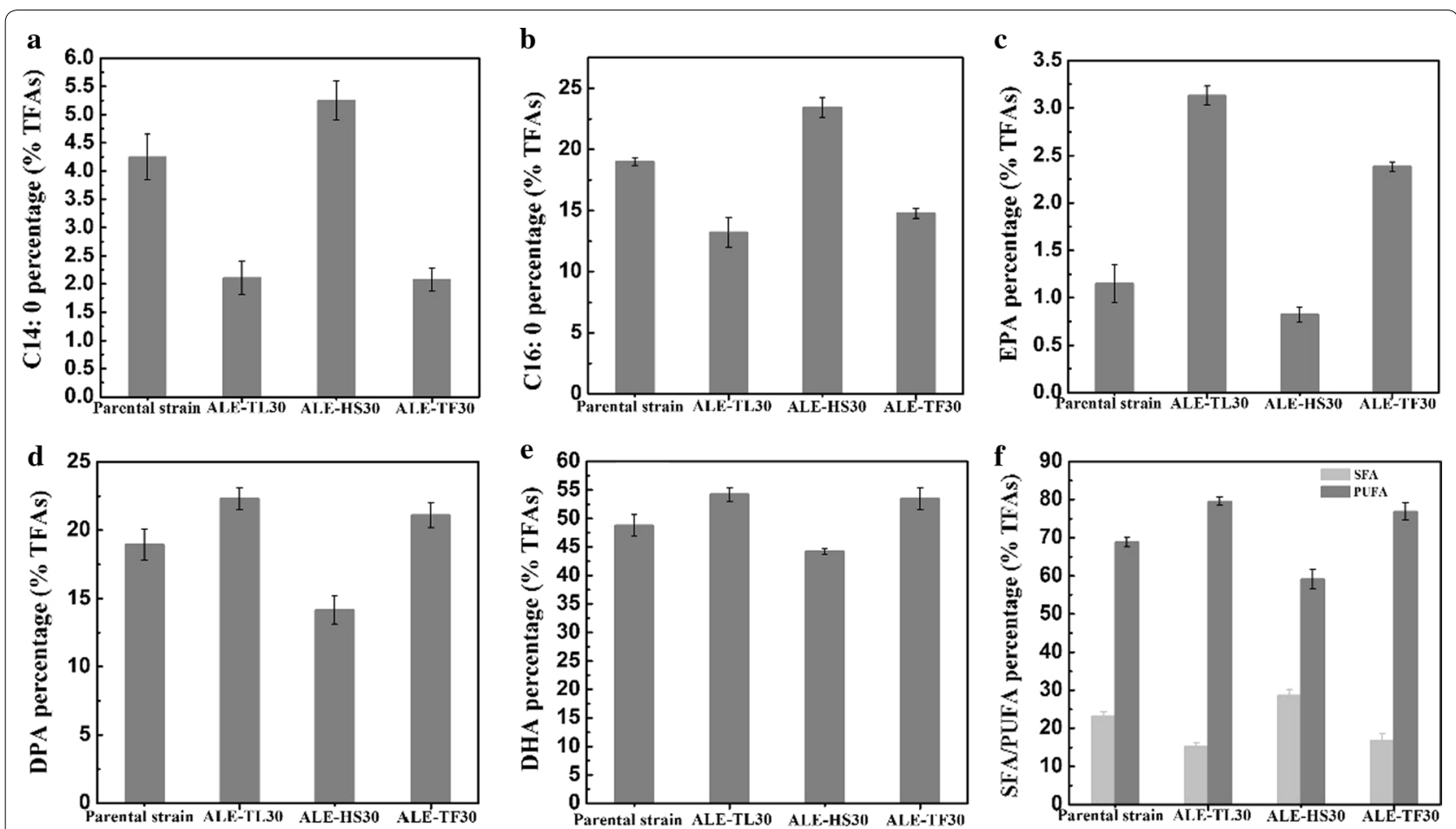

Fig. 2 Fatty acid content (\%TFAs) of the starting strain and evolved strains (ALE-LT30, ALE-HS30 and ALE-TF30) at the end of fermentation. a C14:0 percentage (\% TFAs). b C14:0 percentage (\% TFAs). c EPA percentage (\% TFAs), d DPA percentage (\% TFAs)SFA, e DHA percentage (\% TFAs) content, $\mathbf{f}$ SFA represents the summation of C14:0 and C16:0. PUFA represents the summation of EPA, DPA and DHA. Values and error bars represent the means and the standard deviations of triplicate experiments 


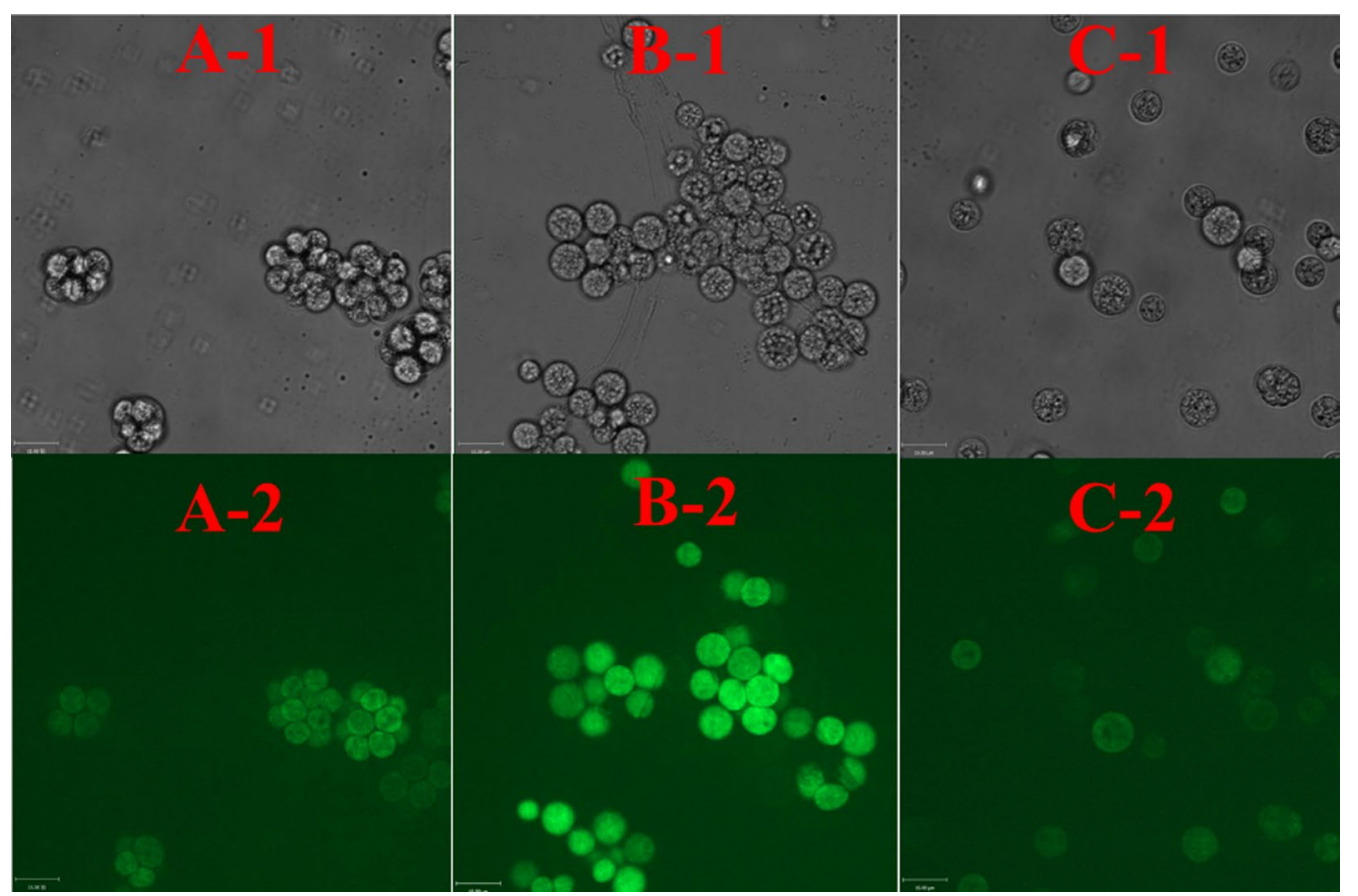

Fig. 3 Determination of cell morphology and ROS levels using DCFH-DA by confocal laser microscopy. A The parental strain cultured in normal medium for 1 cycle. B The parental strain cultured under low-temperature and high-salinity conditions for 1 cycle. CThe evolved strain ALE-TF30 cultured in normal medium for 1 cycle

that were not adapted to environmental stress showed a severely damaged, clustered appearance (Fig. 3B-1), similar to what was observed in Chlamydomonas reinhardtii [21]. Moreover, strong green fluorescence indicated that the cells had suffered severe oxidative damage (Fig. 3B-2), much more than the parental strain (Fig. 3A-2). Interestingly, the ALE-TF30 strain showed signs of abnormal cell division stages, with individual cells found in isolation (Fig. 3C-1), as opposed to the multi-cell clusters of the parental strain (Fig. 3A-1). In addition, with the progress of the adaptive evolution, the cells gradually adapted to the low-temperature and high-salinity conditions, with lower green fluorescence in the strain ALE-TF30 (Fig. 3C-2) demonstrating its improved ROS-scavenging ability or higher antioxidant capacity.

\section{Characterization of the batch-fermentation behavior of the strains evolved via the cooperative two-factor ALE procedure}

To further investigate and increase the ability of the evolved strain ALE-TF30 to produce DHA-rich lipids, we submitted it to conventional culture in a 5 - $\mathrm{L}$ bioreactor. To better analyze the process of cell growth and lipid accumulation and understand the effects of lipid peroxidation on the production of PUFA, and especially DHA, the fermentation process was divided into two stages according to their PUFA accumulation characteristics. Stage I represents the process from the beginning until $84 \mathrm{~h}$, which encompasses the phase of rapid cell growth and lipid accumulation. The PUFA percentage (as \% of TFAs) increased gradually during this stage. Stage II was the oxidative damage phase $(84-120 \mathrm{~h})$ with severe lipid peroxidation and a decline of the PUFA percentage (as \% of TFAs). As shown in Fig. 4a, b, in stage I, the evolved strain ALE-TF30 exhibited better growth and faster consumption of substrates than the parent strain, including the consumption of glucose and MSG. The strain's superiority became more obvious in stage II, which yielded the largest CDW of $126.4 \mathrm{~g} / \mathrm{L}$ by ALE-TF30 at $120 \mathrm{~h}$, representing a $27.2 \%$ increase over the parental strain. After nitrogen exhaustion, the total lipid concentration increased sharply in both strains, from 18.32 and $18.77 \mathrm{~g} / \mathrm{L}$ at $48 \mathrm{~h}$ to 40.12 and $45.23 \mathrm{~g} / \mathrm{L}$ at $84 \mathrm{~h}$, respectively. Nevertheless, the endpoint strain exhibited an overall better lipid productivity in stage II than the starting strain, e.g., $0.73 \mathrm{~g} / \mathrm{L} / \mathrm{h}$ from 84 to $120 \mathrm{~h}$, which represented a $121 \%$ increase over the starting strain (Fig. 4b). The final lipid yield of the evolved strain ALE-TF30 was $71.56 \mathrm{~g} / \mathrm{L}$, which was $37.32 \%$ higher than that of the starting strain (Table 2). This implied that the strain ALETF30, i.e., the final product of the cooperative two-factor evolution possess, had a stronger antioxidant capacity, 

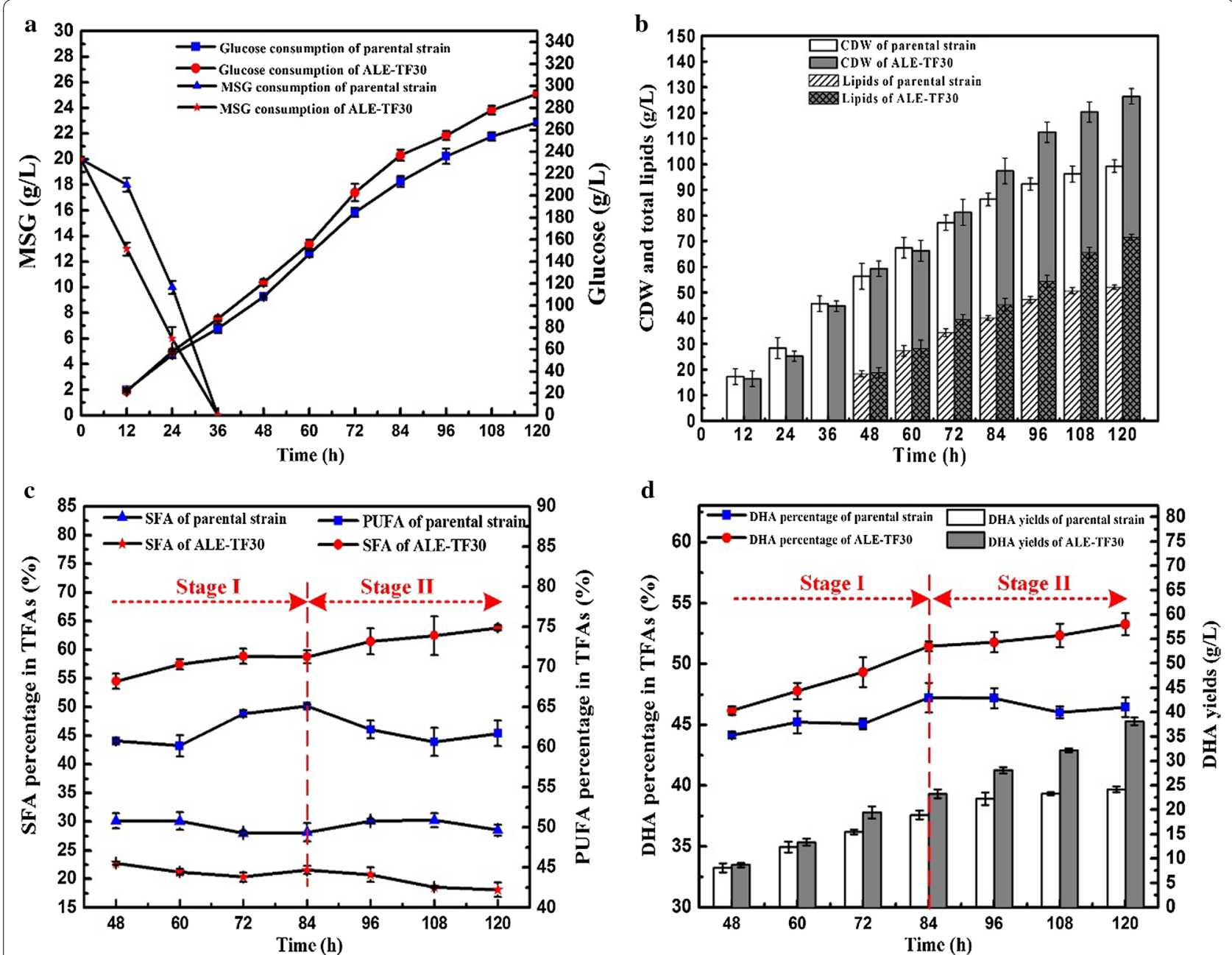

Fig. 4 Comparison between the parental strain and the endpoint strain ALE-TF30 for substrate consumption (a), CDW and lipids (b), SFA and PUFA percentage in TFAs (c), as well as DHA percentage in TFAs and DHA yield (d) in a 5-L bioreactor. Values and error bars represent the means and the standard deviations of triplicate experiments

Table 2 Fermentation parameters of the starting strain and endpoint strains at $120 \mathrm{~h}$ in a 5-L bioreactor

\begin{tabular}{llll}
\hline & Starting strain & $\begin{array}{l}\text { Endpoint } \\
\text { strain } \\
\text { (ALE-TF30) }\end{array}$ & Increase (\%) \\
\hline CDW (g/L) & $99.2 \pm 2.5$ & $126.4 \pm 3.1$ & 27.42 \\
Lipid yield (g/L) & $52.11 \pm 0.8$ & $71.56 \pm 1.2$ & 37.32 \\
$\begin{array}{l}\text { DHA percentage (\% } \\
\text { TFAs) }\end{array}$ & $46.44 \pm 0.8$ & $53.27 \pm 0.9$ & 14.71 \\
$\begin{array}{l}\text { DHA yield (g/L) } \\
\text { DHA productively } \\
\text { (mg/L/h) }\end{array}$ & 24.2 & 38.12 & 57.52 \\
\hline
\end{tabular}

Data represent the mean values and standard deviations of three replicates for each measurement and thus fared better in the oxidative damage stage. In recent years, many efforts have been made to improve biomass and lipid accumulation by substrate optimization. For example, the biomass was increased to $88.6 \mathrm{~g} / \mathrm{L}$ by a MSG-feeding strategy in Schizochytrium sp. LU310 [22], which was favorable for the later lipid accumulation stage. Moreover, when a combination of yeast extract and MSG was used, $55.83 \mathrm{~g} / \mathrm{L}$ of biomass and a lipid productivity of $0.203 \mathrm{~g} / \mathrm{L} / \mathrm{h}$ were obtained in Schizochytrium sp. ATCC 20888 [23].

Similarly, the PUFA and DHA percentages of the parental strain (as \% of TFAs) reached a maximum of $65.11 \%$ and $47.23 \%$ at $84 \mathrm{~h}$, respectively, followed by a decline in stage II (Fig. 4c). By contrast, the strain ALETF30 showed a continuous increase of the PUFA and DHA percentage during the entire fermentation process, with respective maximal values of 74.88 and $53.27 \%$ 
obtained at $120 \mathrm{~h}$. Hence, the highest DHA yield of $38.12 \mathrm{~g} / \mathrm{L}$ was obtained at $120 \mathrm{~h}$ in the endpoint strain, which was $57.52 \%$ higher than that of the parental strain (Fig. 4d, Table 2). These results clearly indicate that cooperative two-factor ALE can effectively promote the production of lipids and DHA, especially at stage II. In addition to ALE, mutagenesis is widely used as a simple and effective breeding strategy to produce mutant strains for DHA hyperproduction. The mutant strain Schizochytrium sp. S1, which was obtained via by low-energy ionizing radiation treatment, showed a higher DHA percentage of $46.2 \%$ [24]. Moreover, in our previous study, a DHA percentage of up to $56.22 \%$ was achieved using $\mathrm{N}$-methyl-N0-nitro- $\mathrm{N}$-nitrosoguanidine induction coupled with ultraviolet mutagenesis [25].

\section{Evaluation of lipid peroxidation}

In order to study the connection between lipid accumulation and oxidative damage, the intracellular amounts of ROS and T-AOC were determined (Fig. 5a, b). Moreover, lipid peroxidation is another commonly used stress marker, which can be measured by determining the malondialdehyde (MDA) content in the cells (Fig. 5c). In both strains, the ROS levels decreased before $48 \mathrm{~h}$, and subsequently dramatically increased from $60 \mathrm{~h}$ until the end of fermentation. Compared with the starting strain, a significant drop in the ROS levels occurred in ALE-TF30, especially after $84 \mathrm{~h}$, leading to a minimum ROS level of 105.7 (fluorescence intensity/g CDW) at $120 \mathrm{~h}$, which was $33.50 \%$ lower than that of the parental strain. Overall, the T-AOC of all cultures decreased with increasing fermentation time. The parental strain maintained a nondetectable level from $60 \mathrm{~h}$ until the end of fermentation, which demonstrates that wild-type Schizochytrium sp. has a serious deficiency of T-AOC, whereby the T-AOC values of the evolved strain were significantly higher and maintained an overall stronger antioxidant capacity during the entire fermentation period. MDA is a major indicator of damage to lipids and increased lipid peroxidation, which indicates severe oxidative stress. The MDA content was increased in both strains after $36 \mathrm{~h}$, but the increase observed in ALE-TF30 was significantly lower than that of the parental strain. These results highlight the stronger antioxidative capacity of the evolved strain, which resulted in a much better defense against lipid peroxidation.

\section{Verification of the expression levels of key enzymes by RT-qPCR}

To validate the metabolic observations, the three key genes associated with oxidative stress (SOD, CAT, and APX), and four key genes of fatty acid biosynthesis (FAS, ORFA, ORFB, ORFC) were analyzed by qRT-PCR at 60 and $108 \mathrm{~h}$ (Fig. 6). In microalgae, a number of biological
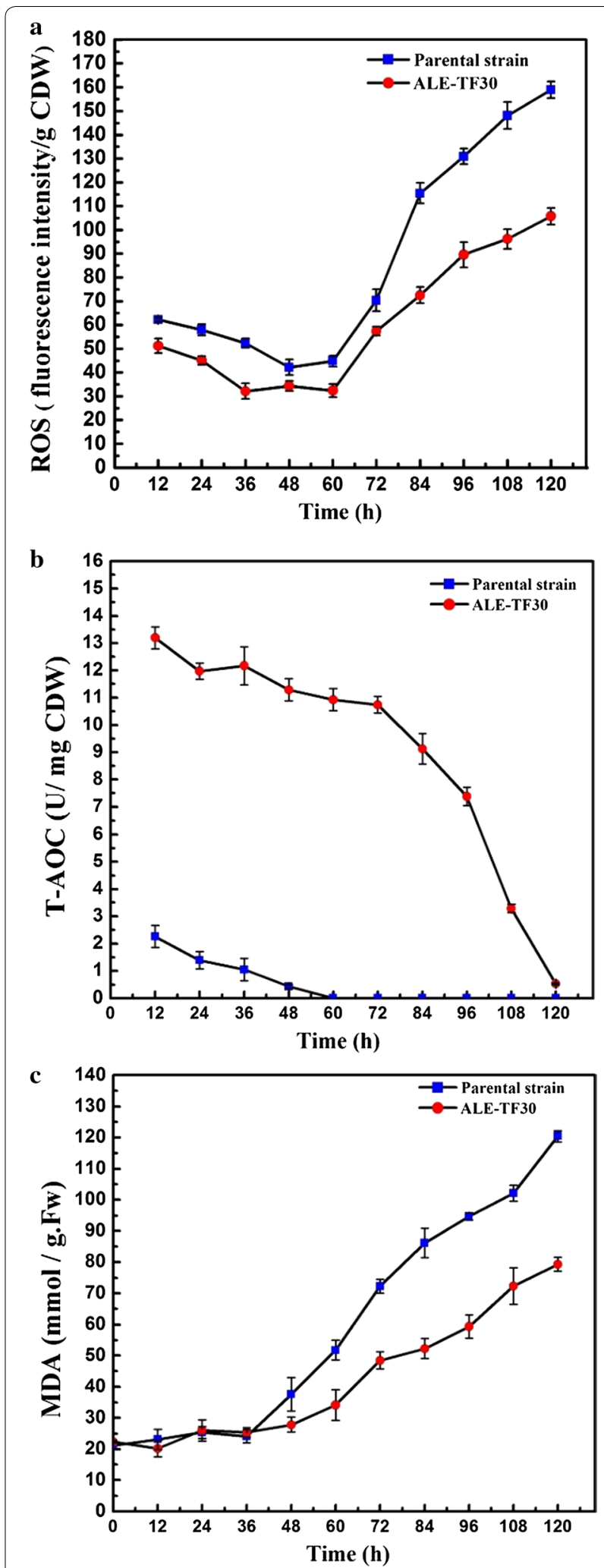

Fig. 5 Comparison between the parental strain and the endpoint strain ALE-TF30 for ROS (a), T-AOC (b), and MDA (c) in a 5-L bioreactor. Values and error bars represent the means and the standard deviations of triplicate experiments 
defense mechanisms have evolved to neutralize ROS, including the action of antioxidant enzymes such as superoxide dismutase (SOD), catalase (CAT), and ascorbate peroxidase (APX) [26]. The steps involving the conversion of the exceedingly toxic superoxide anion $\left(\mathrm{O}_{2}{ }^{-}\right)$ into merely toxic hydrogen peroxide $\left(\mathrm{H}_{2} \mathrm{O}_{2}\right)$ by SOD and the subsequent disproportionation of $\mathrm{H}_{2} \mathrm{O}_{2}$ into innocuous $\mathrm{O}_{2}$ and $\mathrm{H}_{2} \mathrm{O}$ by CAT are very important [27, 28]. In addition, $\mathrm{H}_{2} \mathrm{O}_{2}$ can also be scavenged by APX using ascorbate as a sacrificial substrate [29]. Compared to the parental strain, an overall increase of the gene expression levels of SOD (2.62-fold higher), CAT (2.33-fold higher), and APX (11\% higher) was seen in the ALE-TF30 strain at $60 \mathrm{~h}$. However, as the culture time increased to $108 \mathrm{~h}$, the expression of SOD, CAT, and APX was downregulated in both strains.

In Schizochytrium sp., FAS is a crucial contributor to SFA biosynthesis, and ORFA, ORFB, and ORFC, encoding polyketide synthases, are responsible for the synthesis of LC-PUFAs [30]. As shown in Fig. 6, cooperative twofactor ALE led to a slight increase of FAS gene expression. Interestingly, the ALE-TF30 strain displayed a much higher increase of the gene expression of the PUFA synthase subunits ORFA, ORFB, and ORFC, which were approximately 2.74-, 3.46-, and 4.12-fold higher than in the parental strain at $60 \mathrm{~h}$, respectively. Similarity, the expression of these three genes was downregulated after entering the oxidative damage stage. Nevertheless, the evolved strain maintained significantly stronger expression of ORFA, ORFB, and ORFC (2.30-, 2.92-, 2.74-fold higher than in the parental strain) at $120 \mathrm{~h}$, indicating that the metabolic fluxes towards the FAS and PKS pathways were changed by the evolutionary process.

\section{Discussion}

In this study, a novel cooperative two-factor adaptive laboratory evolution (ALE) protocol was developed to create a stable improved strain that efficiently produces PUFA-rich lipids. In microalgae, temperature stress generally has a greater effect on the lipid profile than on overall lipid production. For instance, the DHA fraction in the TFA of Aurantiochytrium mangrovei increased from 29 to $42 \%$ in the late lipid accumulation phase when the temperature was decreased from 30 to $12{ }^{\circ} \mathrm{C}$ [31]. However, high salinity can stimulate lipid accumulation in microalgae [32], which can be attributed to osmotic stress that may be analogous to nutrient stress regarding the cellular responses [5]. Moreover, Van et al. reported that $\mathrm{NaCl}$ was the most efficient inducer of antioxidant enzymes in microalgae [33]. Unfortunately, the higher accumulation of lipids or PUFA induced via low temperature and high salinity is usually achieved at the expense of lower biomass. Hence, a cooperative two-factor ALE strategy including both low temperature and high

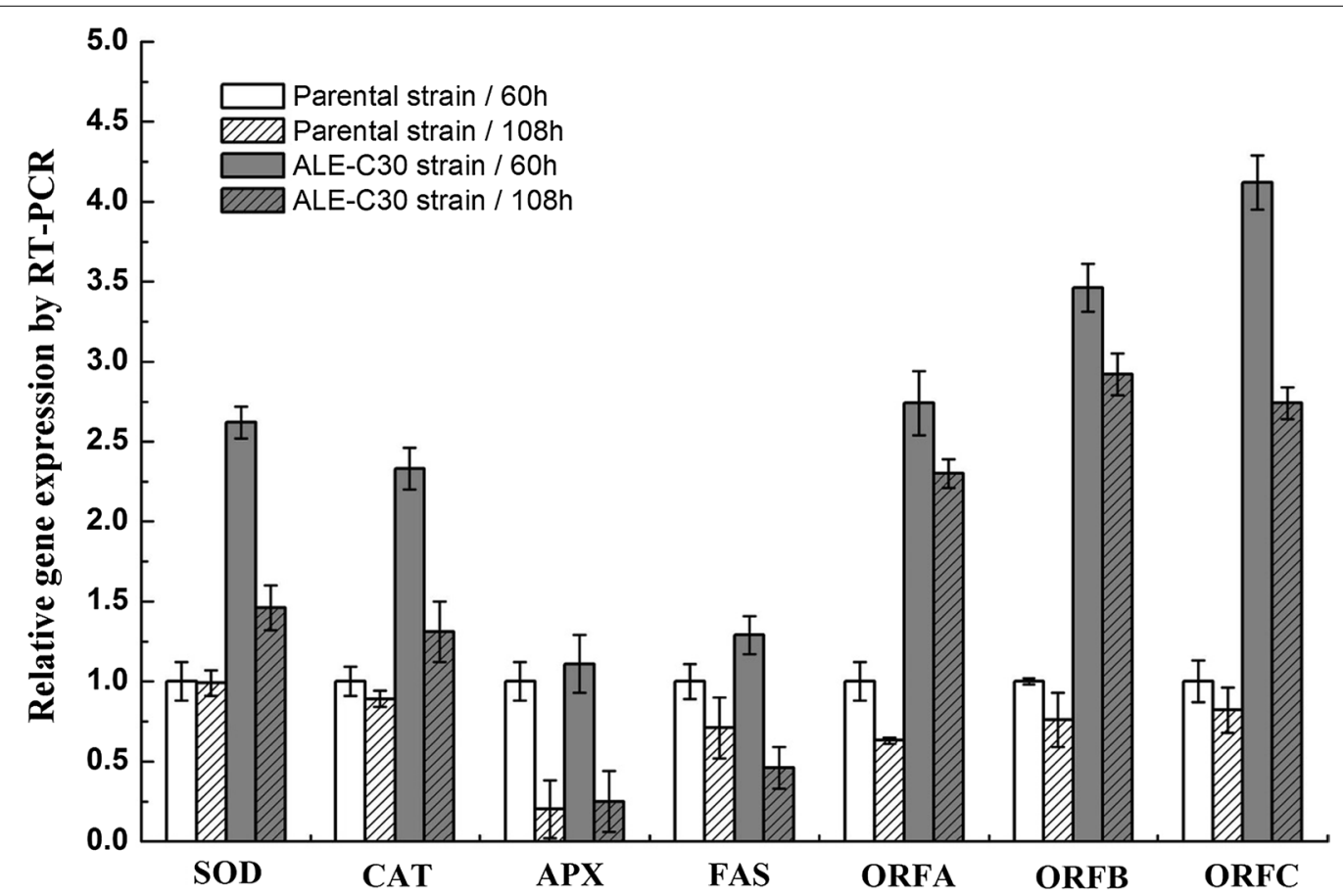

Fig. 6 Real-time quantitative PCR results for the SOD, CAT, APX, FAS, ORFA, ORFB, and ORFC genes from the parental strain and endpoint strain ALE-TF30. Values and error bars represent the means and the standard deviations of triplicate experiments 
salinity was proposed to evolve more robust and promising mutants. On one hand, low-temperature conditions were used to improve the PUFA content. On the other hand, high-salinity conditions were applied to stimulate lipid accumulation and enhance the antioxidative defense systems of Schizochytrium sp. Cooperative two-factor ALE therefore not only increased the accumulation of PUFA-rich lipids, but also prevented the loss of lipids caused by peroxidation.

Under low-temperature conditions, more PUFAs are synthesized and incorporated into the membrane to maintain its fluidity and permeability [34], and the enhancement of PUFA production in cells under temperature-stress conditions has been studied early on by Béchet et al. [35]. A recent proteomics study indicated that cold stress inhibits the cellular supply of energy from glycolysis and the TCA cycle [36], which might be the key reason for the low CDW observed in the low-temperature ALE experiment. However, the increase of EPA percentage was more remarkable than that of DPA or DHA in low-temperature ALE (Figs. 1a, 2c). Many studies suggested that EPA is not required for bulk bilayer fluidity but plays a beneficial role in membrane organization at low temperatures, which appears to rely on special interactions between proteins and EPA-containing phospholipids [37]. In Shewanella livingstonensis, EPA is required for normal membrane organization and cell division at temperatures of around $4{ }^{\circ} \mathrm{C}$ but not at $18{ }^{\circ} \mathrm{C}$ [38]. Thus, the observed increase of EPA percentage during lowtemperature ALE may be partially due to the need to enable the correct expression and folding of membrane proteins under these conditions. By contrast, an increase of the saturated fatty acid proportion was observed under high-salinity stress (Figs. 1b, 2a, b), and the adjustment of membrane permeability needed to avoid an extensive influx of $\mathrm{Na}^{+}$and $\mathrm{Cl}^{-}$ions might explain this response [39]. This trend was also observed in the cells of Dunaliella and Chlamydomonas spp. [40, 41]. Thankfully, lipid accumulation was increased in ALE-HS30 and ALE-TF30 (Table 1), probably due to the need for storing energy-rich compounds to be able to survive in a highsalinity environment [42]. However, further research is needed to elucidate the mechanisms of lipid upregulation under high salinity.

Lipid synthesis can be induced by various stresses related to nutrient availability and environmental factors, among which nitrogen limitation is recognized as the most successful induction strategy, and is most widely used [3]. However, microorganisms tend to accumulate ROS under these conditions [43]. Moreover, a large number of studies have reported that a higher oxygen supply was beneficial for cell growth and lipid accumulation during the cultivation process of Schizochytrium sp.
[44]. Despite the fact that over $90 \%$ of the oxygen consumed by living organisms is used to produce energy for cell growth and lipid accumulation, there always is an inevitable aspect of its conversion into ROS [45]. At the first stage of fermentation, lower levels of ROS did not cause damage to the cells, and there was a sharp increase of CDW and lipids in both strains during this phase (Fig. 4b). Although it has been documented that nitrogen starvation can induce oxidative stress [43], this was not observed in this study. As shown in Fig. 4a, nitrogen (MSG) was depleted at $36 \mathrm{~h}$, but ROS reached their highest value at $12 \mathrm{~h}$ and then gradually declined until $60 \mathrm{~h}$ (Fig. 5a). One reason for the apparent peak in ROS levels at $12 \mathrm{~h}$ might be the stress experienced by the cells when they were shifted from the nutrient-depleted seed culture medium into a nutrient-rich fermentation medium, and this phenomenon was also observed in other microorganisms [43]. The stage spanning $36-60 \mathrm{~h}$ coincides with the exponential growth phase of Schizochytrium sp., during which the cells exhibited strong antioxidant enzyme activity which enabled them to resist the increase of ROS levels induced by nitrogen starvation. However, with the increase of cell concentrations in stage II, growth inhibition and nutritional limitation caused high levels of ROS (Fig. 4a) concomitant with a decrease of cell growth and lipid accumulation. In addition, lipid peroxidation can also lead to the accumulation of high levels of ROS [7]. Johansson et al. established a bio-sustainable PUFA production system in Saccharomyces cerevisiae, and they found that the PUFA-producing strain accumulated high levels of ROS, which resulted in low viability [46]. The sharp increase of ROS in stage II is therefore partly caused by inevitable lipid peroxidation. Furthermore, Fig. 5a, c also show a synchronous trend between the changes of ROS and MDA, a trend that suggests that oxidative stress is a consequence of an increase of lipid peroxidation (indicated by MDA) brought about by ROS.

Schizochytrium sp. only possess one intact pathway containing anaerobic polyketide synthase (PKS) for DHA synthesis, which is distinct from the fatty acid synthase pathway (FAS) [47]. While oxygen limitation strategies lead to an increase of the DHA percentage, they also significantly reduce the biomass productivity, thereby resulting in a decrease of total production and overall productivity. For instance, Jakobsen et al. found that the content of DHA reached $52 \%$ in oxygen limited cells, but CDW decreased to 25-27 from 90 to $100 \mathrm{~g} / \mathrm{L}$ in Aurantiochytrium sp. [44]. In addition to oxygen, various stress conditions or lipid peroxidation can also induce ROS generation. However, Schizochytrium sp. has a weak ability to remove ROS, which was evidenced by the T-AOC of the parental strain, which decreased to non-detectable levels at $60 \mathrm{~h}$ and remained there until 
the end of fermentation (Fig. 5b). The resulting lipid peroxidation can cause a loss of the produced lipids, which might explain the decrease of the DHA and PUFA percentages in the parental strain after $84 \mathrm{~h}$ (Fig. 4c, d). Although the PUFA content of ALE-TF30 increased less and even decreased after $84 \mathrm{~h}, \mathrm{DHA}$ is more recalcitrant to oxidation than DPA because the $\Delta 4$ double bond must first be removed by peroxisomal $\beta$-oxidation [48]. Thus, an apparent selective retention of DHA resulted in a continuous increase of the percentage of DHA in TFAs until the end of fermentation. As a consequence, maintenance of ROS detoxification is paramount to the survival of all aerobic life forms. In recent years, an increasing amount of work was done on strategies to scavenge ROS or enhance T-AOC by adding antioxidants to improve microalgal performance. For example, DHA productivity was increased by 20 and 44\% in Crypthecodinium cohnii and Schizochytrium sp. by adding sesamol and ascorbic acid, respectively $[9,49]$. In addition, Gaffney et al. improved the T-AOC of Schizochytrium sp. via the supplementation of flaxseed oil [50]. Moreover, increasing experimental evidence seems to point in the direction that intracellular ROS may in fact be general mediators of lipid accumulation in oleaginous microorganisms [51]. In fact, many researchers attributed the stress-mediated induction of lipid accumulation to the generation of ROS [52]. Kang et al. used oxidative stress to induce lipid production in Chlorella vulgaris [53]. Similarly, Yilanciooglu et al. applied exogenous $\mathrm{H}_{2} \mathrm{O}_{2}$, which resulted in oxidative stress and increased the cellular lipid content up to $44 \%$ [54]. However, only a few potential mechanisms of ROS-mediated lipid accumulation have been discovered, and direct experimental evidence is still lacking.

In addition to ROS, microalgae have also evolved adaptive ways to combat salt stress, adjustment of the expression of antioxidant enzymes being one such adaptive mechanism, which is potentially more attractive due to its impact on lipid production. Tang et al. reported that enhanced SOD and CAT activity provides protection to Scytonema javanicum by scavenging ROS, thereby mitigating the oxidative burst caused by $\mathrm{NaCl}$ stress [55]. Likewise, exposing cells to high doses of $\mathrm{NaCl}$ led to a significant rise in the activities of SOD, POD, CAT, and GST in Nostoc muscorum and Phormidium foveolarum [56]. In the present study, the most striking observation was the overall increase of T-AOC in the ALE-TF30 strain (Fig. 5b). To further explore these results, the gene expression of SOD, CAT, and APX was measured at 60 and $108 \mathrm{~h}$. Oxidative stress generally involves increases in the production of $\mathrm{O}_{2}{ }^{-}$ and $\mathrm{H}_{2} \mathrm{O}_{2}$. In the evolved strain, the gene expression of SOD and CAT was sharply increased, whereas APX showed only 1.11 and $38 \%$ increases over the parent strain at 60 and $108 \mathrm{~h}$, respectively, which indicated that the high T-AOC in ALE-TF30 was mostly attributable to SOD and CAT. In contrast to APX, SOD and CAT act as the first line of defense against oxidative damage, and are crucial for the detoxification of $\mathrm{O}_{2}{ }^{-}$ and $\mathrm{H}_{2} \mathrm{O}_{2}$ [57], which might explain the lower increase of gene expression of APX in the evolved strain. During the later stages of fermentation, high ROS levels due to nitrogen limitation, growth inhibition, and strong lipid peroxidation may have caused damage to proteins, resulting in lower gene expression or loss of function of antioxidant enzymes. This may be the reason why the genes encoding fatty acid biosynthesis enzymes and antioxidant enzymes were all downregulated in the two strains during the oxidative damage stage (108 h; Fig. 6). This phenomenon was also observed in other microorganisms [55].

The ultimate aim of this study was to develop a strain with high DHA production capacity, which is why key genes associated with fatty acid biosynthesis-FAS, ORFA, ORFB, and ORFC-were also analyzed. The expression of PUFA synthases (ORFA/B/C) was remarkably increased in the evolved strain, which was in agreement with other studies on the effects of low-temperature stress [58]. However, the dramatic rise in the expression of PUFA-related genes resulted in only a moderate increase of PUFA production in the ALE-TF30 strain, which might indicate that the overexpression of polyketide synthases outpaced the cells' supply of energy or precursors for PUFA synthesis. This was in agreement with many studies which showed that genes related to lipid biosynthesis, such as acetyl-CoA carboxylase and acyl-CoA synthetase, were significant upregulated under conditions of environmental stress $[59,60]$. Among the three genes encoding PKS enzymes, ORFC expression was increased the most, and was 4.12- and 3.34-fold higher than that of the parental strain at 60 and $180 \mathrm{~h}$, respectively. ORFC contains two DH (dehydrase) domains and one ER (enoyl-ACP reductase) domain, which showed higher sensitivity to the changes of environmental factors [61]. $\mathrm{DH}$ catalyzes the conversion of ketoacyl-ACP to enoylACP, and the ER domain catalyzes the final step of fatty acid chain extension in which it reduces the double bond. The omission of enoyl-ACP reduction determines the formation of the double bonds of PUFAs. Inspired by this, Cheng et al. developed a method for screening Aurantiochytrium sp. strains with high DHA yield using inhibitors of enoyl-ACP reductase and synergistic usage of cold stress at $4{ }^{\circ} \mathrm{C}$ [62]. The expression level of ORFC might thus explain the changes of fatty acid composition during DHA fermentation. 


\section{Conclusions}

To our best knowledge, this is the first comprehensive study to take into account both lipid overproduction and lipid peroxidation in the fermentation process. We explored the cooperative effect of low temperature and high salinity in ALE on the growth and DHA production of Schizochytrium sp. The best obtained strain, ALETF30, displayed both a good DHA synthesis ability and strong antioxidant capacity. The maximum DHA yield with minimum lipid peroxidation was obtained by the endpoint strain of the evolution process. These major performance changes were accompanied by an overall increase of gene expression levels of antioxidant enzymes and polyketide synthases. Such knowledge provides a new method that can be used in other oil-producing microalgae, and proves that ALE is an effective strategy to generate high-performing strains of Schizochytrium sp. for industrial applications.

\section{Methods}

\section{Microorganism and culture conditions}

Schizochytrium sp. HX-308 (CCTCC M 209059), isolated from seawater and stored in the China Center for Type Culture Collection (CCTCC), was used in this study. The strain was preserved in $20 \%(\mathrm{v} / \mathrm{v})$ glycerol at $-80{ }^{\circ} \mathrm{C}$.

The seed culture medium and conditions of propagation were the same as those used in our previous study [63]. After three generations, the seed cultures ( $1 \% \mathrm{v} / \mathrm{v})$ were transferred to $500-\mathrm{mL}$ shake flasks containing $100 \mathrm{~mL}$ of medium and cultured at $30{ }^{\circ} \mathrm{C}$ under constant orbital shaking at $170 \mathrm{rpm}$, and the resulting cell suspension was used as inoculant for the fermentation. The fermentation medium was the same as reported in our previous study [47]. The basic medium contained $50 \mathrm{~g} / \mathrm{L}$ glucose and $0.4 \mathrm{~g} / \mathrm{L}$ yeast extract, which were dissolved in artificial sea water. The artificial sea water contained (g/L): $\mathrm{Na}_{2} \mathrm{SO}_{4}$ 10, $\left(\mathrm{NH}_{4}\right)_{2} \mathrm{SO}_{4} 0.8, \mathrm{KH}_{2} \mathrm{PO}_{4}$ 4, $\mathrm{KCl} 0.2, \mathrm{MgSO}_{4} 2$, monosodium glutamate 20, $\mathrm{CaCl}_{2}$ 0.1 , and the trace elements solution $(\mathrm{g} / \mathrm{L}): \mathrm{Na}_{2}$ EDTA 6 , $\mathrm{FeSO}_{4}$ 0.29, $\mathrm{MnCl}_{2} \cdot 4 \mathrm{H}_{2} \mathrm{O} 0.86, \mathrm{ZnSO}_{4} 0.8, \mathrm{CoCl}_{2} \cdot 6 \mathrm{H}_{2} \mathrm{O}$ $0.01, \mathrm{Na}_{2} \mathrm{MoO}_{4} \cdot 2 \mathrm{H}_{2} \mathrm{O} \quad 0.01, \quad \mathrm{NiSO} 4 \cdot 6 \mathrm{H}_{2} \mathrm{O} \quad 0.06$, and $\mathrm{CuSO}_{4} \cdot 5 \mathrm{H}_{2} \mathrm{O} 0.6$.

\section{Adaptive laboratory evolution experiments}

ALE was based on a long-term serial transfer procedure using $4{ }^{\circ} \mathrm{C}$ and $30 \mathrm{~g} / \mathrm{L} \mathrm{NaCl}$ as low-temperature and highsalinity stress inducers, respectively. The experimental process was conducted as shown in Fig. 7. For each new cycle, the seed culture $(1 \% \mathrm{v} / \mathrm{v})$ was transferred to fresh medium. In addition to the stress inducer, the culture medium or condition of the evolved strain was the same as for the ancestral strain. Every $24 \mathrm{~h}$, the seed culture
$(1 \% \mathrm{v} / \mathrm{v})$ was transferred to a fresh medium. The method of cooperative two-factor ALE is similar to low-temperature ALE, the only difference being high-salinity medium. In this study, every $72 \mathrm{~h}$ was defined as one cycle, and three ALE experiments were conducted repeatedly for 30 cycles. After 30 cycles, low-temperature ALE, high-salinity ALE, and the cooperative two-factor ALE strains were named ALE-LT30, ALE-HS30, and ALE-TF30, respectively. The ALE-TF30 strain was the endpoint strain. A serial transfer experiment has proved that the endpoint strain ALE-TF30 had a fixed stable improved phenotype. The fermentation medium was the same as in our previous study [47]. All fermentation experiments were performed in triplicate. Such serial transfers were repeated for 30 cycles and $1 \mathrm{~mL}$ samples of the evolving population were taken and stored at $-80{ }^{\circ} \mathrm{C}$ in $20 \%$ glycerol for subsequent analysis every 5 cycles.

\section{Determination of cell dry weight and total lipids}

Cell dry weight was determined gravimetrically by harvesting $10 \mathrm{~mL}$ culture aliquots by centrifugation at $4500 \times g$ for $5 \mathrm{~min}$. The cells were subsequently transferred to a weighed filter paper and dried at $60{ }^{\circ} \mathrm{C}$ to constant weight $(\sim 12 \mathrm{~h})$. The yield of total lipids, extracellular glucose, and glutamate were determined the same way as published in our previous study [63].

\section{Fatty acid analysis}

Fatty acid methyl esters (FAMEs) were prepared from $0.2 \mathrm{~g}$ of dried cells and analyzed using a GC-2010 gas chromatography system (Shimadzu, Japan), equipped with a DB-23 capillary column $(60 \mathrm{~m} \times 0.22 \mathrm{~mm}$; Agilent, USA) and flame ionization detector (FID). Nitrogen was used as the carrier gas. The injector was maintained at $250{ }^{\circ} \mathrm{C}$ with an injection volume of $1 \mu \mathrm{L}$. The column temperature was raised from 100 to $200{ }^{\circ} \mathrm{C}$ at $25{ }^{\circ} \mathrm{C} /$ min and then increased to $230{ }^{\circ} \mathrm{C}$ at $4{ }^{\circ} \mathrm{C} / \mathrm{min}$, followed by keeping this temperature for $9 \mathrm{~min}$. The FID detector temperature was set to $280{ }^{\circ} \mathrm{C}$. FAMEs were identified by comparing the retention times with corresponding external authentic reference standards (Sigma, USA). The quantities of individual FAMEs were estimated based on the integrated peak areas on the chromatogram using non-adecanoic acid (C19:0) as the internal standard.

\section{Determination of total antioxidant capacity}

Intracellular total antioxidant capacity (T-AOC) was determined using the T-AOC assay kit (Angle Gene, China) according to the manufacturer's instructions. Cells were harvested by centrifugation for $5 \mathrm{~min}$ and washed with physiological saline in order to remove the excess of ascorbic acid in the medium. After being disrupted using an ultrasonic disrupter for $15 \mathrm{~min}, 200 \mu \mathrm{L}$ 


\section{Low temperature ALE}
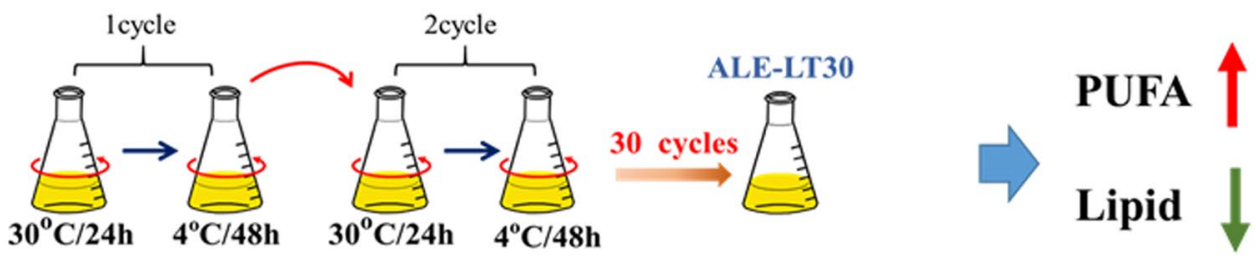

\section{High salinity ALE}
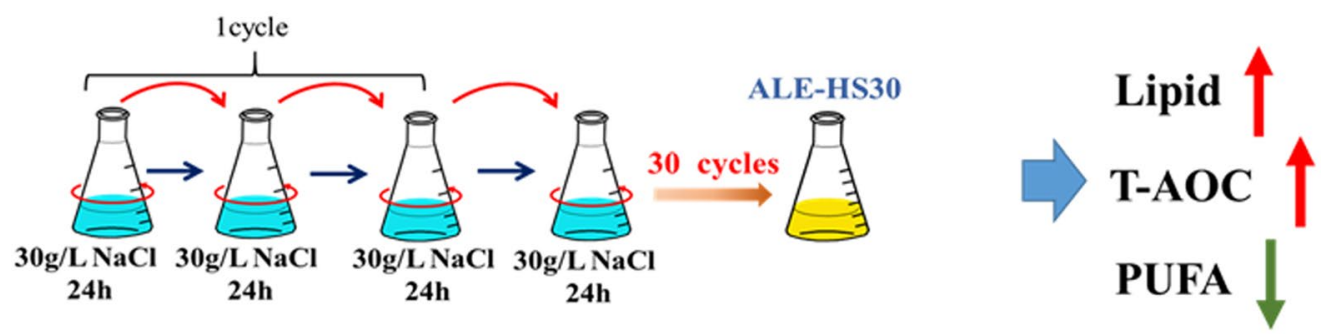

\section{The cooperative two-factor ALE}
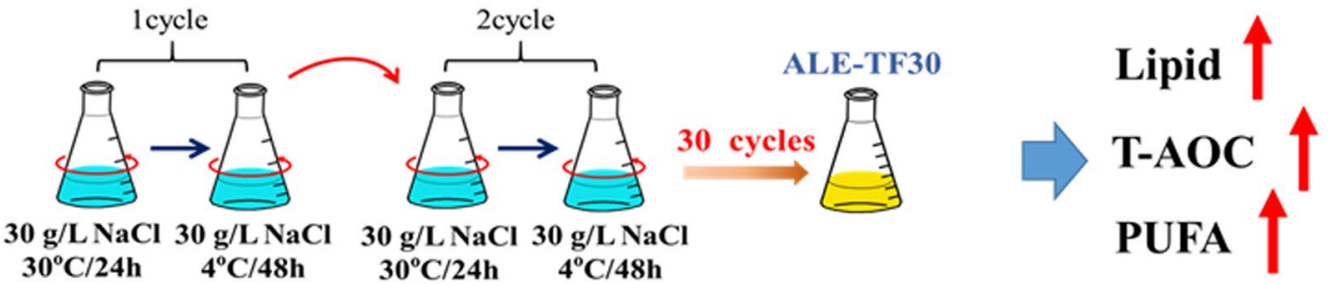

Fig. $\mathbf{7}$ Schematic diagram of the experimental process of the two different adaptive laboratory evolution (ALE) strategies. Yellow represents normal medium and blue represents high-salinity medium

of crude extract was mixed with $0.2 \mathrm{mM}$ DPPH radical. The mixture was incubated for $30 \mathrm{~min}$ at $37{ }^{\circ} \mathrm{C}$ and the absorbance at $517 \mathrm{~nm}$ was measured. The radical scavenging ability was calculated based on the difference in the measured decrease of absorbance at $517 \mathrm{~nm}$ between the blank and the respective sample, whereby a unit of $\mathrm{T}$-AOC was defined as the increase of $\mathrm{OD}_{517}$ per mg cell dry weight, per minute, at $37^{\circ} \mathrm{C}$. The value of T-AOC was calculated using the following equation:

$$
\mathrm{T}-\mathrm{AOC}(\mathrm{U} / \mathrm{mg} \mathrm{CDW})=\frac{\frac{\mathrm{Ar}-\mathrm{Ac}}{0.01 * 30}}{\mathrm{CDW}}
$$

where $\mathrm{Ar}$ is the absorbance of the reaction sample (with $\mathrm{DPPH}$ solution) and Ac is the absorbance of the control sample (without DPPH solution).

\section{Determination of intracellular reactive oxygen species}

Intracellular ROS levels were determined according to the method by Li et al. which uses the oxidation-sensitive fluorescent indicator $2^{\prime}, 7^{\prime}$-dichlorofluorescein diacetate (DCFH-DA) [64]. This probe is not fluorescent in its original form and can freely cross cell membranes. Inside the living cells, two acetate groups (DA) are removed from the indicator forming DCFH, which is still not fluorescent. In the presence of ROS, DCFH is oxidized to fluorescent $2^{\prime}, 7^{\prime}$-dichlorofluorescein (DCF), which can be measured by fluorometry. DCFH-DA was added at an in-well concentration of $10 \mathrm{Mm}$ to collected cells, and incubated for $30 \mathrm{~min}$ at $37^{\circ} \mathrm{C}$. After a full half-hour of exposure to the probe, the excess of indicator in the medium was washed with PBS to make sure that only the intracellular oxidation was measured. Fluorescence intensity was measured using a fluorescence spectrophotometer at an excitation wavelength of $485 \mathrm{~nm}$ and an emission wavelength of $530 \mathrm{~nm}$. 


\section{Measurement of lipid peroxidation}

The level of lipid peroxidation was determined by measuring malondialdehyde (MDA) equivalents according to the method of Heath et al. [65]. Treated and untreated cells were homogenized in $5 \%(\mathrm{w} / \mathrm{v})$ TCA and centrifuged at $10,000 \times g$ for $10 \mathrm{~min}$ at $4{ }^{\circ} \mathrm{C}$. The reaction mixture containing thiobarbituric acid-TCA solution and cell extract was boiled for $20 \mathrm{~min}$. The absorbance of the reaction mixture was recorded at 532 and $600 \mathrm{~nm}$. The values of non-specific absorption recorded at $600 \mathrm{~nm}$ were subtracted from the values recorded at $532 \mathrm{~nm}$. The content of MDA equivalents was determined using an extinction coefficient of $155 \mathrm{mM} / \mathrm{cm}$.

\section{Real-time quantitative PCR}

Samples of the parental strain and the ALE-TF30 strain were obtained at 60 and $108 \mathrm{~h}$ of fermentation, and RNA was isolated using the Rapid fungal RNA extraction kit (Zoonbio Biotechnology, Nanjing, China). cDNA was obtained using the TURE script cDNA Synthesis Kit (Zoonbio Biotechnology, Nanjing, China) and used for quantitative PCR analysis. Seven target genes (superoxide dismutase, catalase, ascorbate peroxidase, FAS, ORFA, ORFB, and ORFC) were tested in the present study. The relative levels of the amplified mRNAs were evaluated according to the $2^{-\triangle \triangle \mathrm{Ct}}$ method using $\beta$-actin for normalization.

\section{Additional file}

Additional file 1: Table S1. Endpoint strain of ALE-TF30 was cultured and passaged to sixteen generations at $170 \mathrm{rpm}$ and $30^{\circ} \mathrm{C}$.

\section{Abbreviations}

ALE: adaptive laboratory evolution; APX: ascorbate peroxidase; CAT: catalase; CDW: cell dry weight; DHA: docosahexaenoic acid; DPA: docosapentaenoic acid: EPA: eicosapentaenoic acid; GSH: glutathione; PUFA: polyunsaturated fatty acid; ROS: reactive oxygen species; T-AOC: total antioxidant capacity; TAG : triacylglycerol; TFAs: total fatty acids; TLs: total lipids; MDA: malondialdehyde; SFA: saturated fatty acid; SOD: superoxide dismutase.

\section{Authors' contributions}

XMS conceived the idea, conducted all the experiments, and wrote the first draft of the manuscript. ZQB and XJJ participated in fermentation experiments. JL drafted the figures and tables. $L \mathrm{R}, \mathrm{QYZ}$, and $\mathrm{HH}$ contributed to manuscript revision and approved the final version. All authors read and approved the final manuscript.

\footnotetext{
Author details

${ }^{1}$ College of Biotechnology and Pharmaceutical Engineering, Nanjing Tech University, No. 30 South Puzhu Road, Nanjing 211816, People's Republic of China. ${ }^{2}$ School of Pharmaceutical Sciences, Nanjing Tech University, No. 30 South Puzhu Road, Nanjing 211816, People's Republic of China. ${ }^{3}$ State Key Laboratory of Materials-Oriented Chemical Engineering, Nanjing Tech University, No. 5 Xinmofan Road, Nanjing 210009, People's Republic of China. ${ }^{4}$ Jiangsu National Synergetic Innovation Center for Advanced Materials (SICAM), Nanjing Tech University, No. 5 Xinmofan Road, Nanjing 210009, People's Republic of China.
}

\section{Acknowledgements}

This work was financially supported by the National Science Foundation for Distinguished Young Scholars of China (No. 21225626), the National Natural Science Foundation of China (No. 21306085), the National High Technology Research and Development Program of China (No. 2014AA021701), the Outstanding Youth Foundation of Jiangsu Nature Science Foundation (BK20160092), the Program for Innovative Research Team in University of Jiangsu Province (2015), and the Jiangsu Synergetic Innovation Center for Advanced Bio-Manufacture.

Ethics approval and consent to participate

Not applicable.

\section{Competing interests}

The authors declare that they have no competing interests.

\section{Publisher's Note}

Springer Nature remains neutral with regard to jurisdictional claims in published maps and institutional affiliations.

Received: 11 December 2017 Accepted: 2 March 2018

Published online: 14 March 2018

\section{References}

1. Ursin VM. Modification of plant lipids for human health: development of functional land-based omega-3 fatty acids. J Nutr. 2003;133:4271-4.

2. Tacon AGJ, Metian M. Global overview on the use of fish meal and fish oil in industrially compounded aquafeeds: trends and future prospects. Aquaculture. 2008;285:146-58.

3. Patil KP, Gogate PR. Improved synthesis of docosahexaenoic acid (dha) using schizochytrium limacinum sr21 and sustainable media. Chem Eng J. 2015;268:187-96.

4. Lari Z, Moradi-Kheibari N, Ahmadzadeh H, Abrishamchi P, Moheimani NR, Murry MA. Bioprocess engineering of microalgae to optimize lipid production through nutrient management. J Appl Phycol. 2016;28:3235-50.

5. Duan X. Salt-induced osmotic stress for lipid overproduction in batch culture of chlorella vulgaris. Afr J Biotechnol. 2014;11:7072-8.

6. Río ED, Acién FG, García-Malea MC, Rivas J, Molina-Grima E, Guerrero MG. Efficiency assessment of the one-step production of astaxanthin by the microalga haematococcus pluvialis. Biotechnol Bioeng. 2010;100:397-402.

7. Ruenwai R, Neiss A, Laoteng K, Vongsangnak W, Dalfard AB, Cheevadhanarak $S$, et al. Heterologous production of polyunsaturated fatty acids in saccharomyces cerevisiae causes a global transcriptional response resulting in reduced proteasomal activity and increased oxidative stress. Biotechnol J. 2015;6:343-56.

8. Cipak A, Hasslacher M, Tehlivets O, Collinson EJ, Zivkovic M, Matijevic $T$, et al. Saccharomyces cerevisiae strain expressing a plant fatty acid desaturase produces polyunsaturated fatty acids and is susceptible to oxidative stress induced by lipid peroxidation. Free Radical Bio Med. 2006:40:897-906

9. Ren LJ, Sun XM, Ji XJ, Chen SL, Guo DS, Huang H. Enhancement of docosahexaenoic acid synthesis by manipulation of antioxidant capacity and prevention of oxidative damage in schizochytrium sp. Bioresour Technol. 2016:223:141-8.

10. Grossmann M, Kießling F, Singer J, Schoeman H, Schröder MB, Wallbrunn CV. Genetically modified wine yeasts and risk assessment studies covering different steps within the wine making process. Ann Microbiol. 2011;61:103-15.

11. Dragosits M, Mattanovich D. Adaptive laboratory evolution—principles and applications for biotechnology. Microb Cell Fact. 2013;12:64.

12. Winkler J, Reyes $L H$, Kao KC. Adaptive laboratory evolution for strain engineering. Methods Mol Biol. 2013;985:211-22.

13. Li X, Pei G, Liu L, Lei C, Zhang W. Metabolomic analysis and lipid accumulation in a glucose tolerant crypthecodinium cohnii strain obtained by adaptive laboratory evolution. Bioresour Technol. 2017;235:87-95. 
14. Kazuhiko K, Andreas R, Plassmeier JK, Sinskey AJ. Improved glycerol utilization by a triacylglycerol-producing Rhodococcus opacus strain for renewable fuels. Biotechnol Biofuels. 2015;8:1-11.

15. Sun XM, Ren $\sqcup$, Ji XJ, Chen SL, Guo DS, Huang H. Adaptive evolution of Schizochytrium sp. by continuous high oxygen stimulations to enhance docosahexaenoic acid synthesis. Bioresour Technol. 2016;211:374-81.

16. Tillich UM, Wolter N, Franke P, Dühring U, Frohme M. Screening and genetic characterization of thermo-tolerant Synechocystis sp. Pcc6803 strains created by adaptive evolution. BMC Biotechnol. 2014;14:1-15.

17. Perrineau MM, Zelzion E, Gross J, Price DC, Boyd J, Bhattacharya D. Evolution of salt tolerance in a laboratory reared population of Chlamydomonas reinhardtii. Environ Microbiol. 2014;16:1755-66.

18. Tatsuzawa H, Takizawa E. Changes in lipid and fatty acid composition of Pavlova lutheri. Phytochemistry. 1995;40:397-400.

19. Wagenen JV, Miller TW, Hobbs S, Hook P, Crowe B, Huesemann M. Effects of light and temperature on fatty acid production in nannochloropsis salina. Energies. 2012;5:731-40.

20. Jahnke LS, White AL. Long-term hyposaline and hypersaline stresses produce distinct antioxidant responses in the marine alga Dunaliella tertiolecta. J Plant Physiol. 2003;160:1193-202.

21. Hounslow E, Kapoore RV, Vaidyanathan S, Gilmour DJ, Wright PC. The search for a lipid trigger: the effect of salt stress on the lipid profile of the model microalgal species Chlamydomonas reinhardtii for biofuels production. Curr Biotechnol. 2016:5:305-13.

22. Ling $X$, Guo J, Liu X, Zhang X, Wang N, Lu Y, Ng IS. Impact of carbon and nitrogen feeding strategy on high production of biomass and docosahexaenoic acid (dha) by Schizochytrium sp. Lu310. Bioresour Technol. 2015;184:139-47.

23. Jiang $X$, Zhang J, Zhao J, et al. Regulation of lipid accumulation in Schizochytrium sp. ATCC 20888 in response to different nitrogen sources. Eur J Lipid Sci Technol. 2017;119(10):e201700025. https://doi. org/10.1002/ejlt.201700025.

24. Fu J, Chen T, Lu H, Lin Y, Xie X, Tian H, et al. Enhancement of docosahexaenoic acid production by low-energy ion implantation coupled with screening method based on sudan black b staining in Schizochytrium sp. Bioresour Technol. 2016;221:405-11.

25. Lian M, Huang H, Ren L, Ji X, Zhu J, Jin L. Increase of docosahexaenoic acid production by Schizochytrium sp. through mutagenesis and enzyme assay. Appl Biochem Biotechnol. 2010;162:935-41.

26. Harris ED. Regulation of antioxidant enzymes. J Nutr. 1992;122:2675-83.

27. Imlay JA. Cellular defenses against superoxide and hydrogen peroxide. Annu Rev Biochem. 2008;77:755-76.

28. Li T, Huang X, Zhou R, Liu Y, Li B, Nomura C, Zhao J. Differential expression and localization of $\mathrm{mn}$ and fe superoxide dismutases in the heterocystous Cyanobacterium anabaena sp. strain pcc 7120. J Bacteriol. 2002;184:5096-103.

29. Nakano Y, Asada K. Hydrogen peroxide is scavenged by ascorbatespecific peroxidase in spinach chloroplasts. Plant Cell Physiol. 1981;22:867-80.

30. Metz JG, Roessler P, Facciotti D, Levering C, Dittrich F, Lassner M, et al. Production of polyunsaturated fatty acids by polyketide synthases in both prokaryotes and eukaryotes. Science. 2001;293:290-3.

31. Kanokwan C, Cornelis V. Growth, fatty acid profile in major lipid classes and lipid fluidity of Aurantiochytrium mangrovei sk-02 as a function of growth temperature. Braz J Microbiol. 2012;43:187-200.

32. Takagi M, Yoshida T. Effect of salt concentration on intracellular accumulation of lipids and triacylglyceride in marine microalgae Dunaliella cells. J Biosci Bioeng. 2006;101(3):223-6.

33. van Melle JP, De JP, Mp VDB, Pot HJ, van Veldhuisen DJ. Effect of abiotic stress on photosynthesis and respiration in Chlamydomonas reinhardtii. Induction of oxidative stress. Enzyme Microb Technol. 2006;40:163-7.

34. Jiang $\mathrm{H}$, Gao K. Effects of lowering temperature during culture on the production of polyunsaturated fatty acids in the marine diatom Phaeodactylum tricornutum (bacillariophyceae). J Phycol. 2010;40:651-4.

35. Béchet Q, Shilton A, Guieysse B. Modeling the effects of light and temperature on algae growth: state of the art and critical assessment for productivity prediction during outdoor cultivation. Biotechnol Adv. 2013;31:1648-63.

36. Ma Z, Tian M, Tan Y, Cui G, Feng Y, Cui Q, Song X. Response mechanism of the docosahexaenoic acid producer aurantiochytrium under cold stress. Algal Res. 2017;25:191-9.
37. Kawamoto J, Kurihara T, Kitagawa M, Kato I, Esaki N. Proteomic studies of an antarctic cold-adapted bacterium, Shewanella livingstonensis ac10, for global identification of cold-inducible proteins. Extremophiles. 2007;11:819-26.

38. Kawamoto J, Kurihara T, Yamamoto K, Nagayasu M, Tani Y, Mihara $\mathrm{H}$, et al. Eicosapentaenoic acid plays a beneficial role in membrane organization and cell division of a cold-adapted bacterium, Shewanella livingstonensis ac10. J Bacteriol. 2009;191:632-40.

39. Allakhverdiev SI, Sakamoto A, Nishiyama Y, Murata N. Ionic and osmotic effects of NaCl-induced inactivation of photosystems I and II in Synechococcus sp. Plant Physiol. 2000;123:1047-56.

40. Wang GC, Wang XQ, et al. Acclimatory responses to high-salt stress in Chlamydomonas (chlorophyta, chlorophyceae) from antarctica. Acta Oceanol Sin. 2012;31:116-24.

41. Xu XQ, Beardall J. Effect of salinity on fatty acid composition of a green microalga from an antarctic hypersaline lake. Phytochemistry. 1997:45:655-8.

42. Schüler LM, Schulze PSC, Pereira H, Barreira L, León R, Varela J. Trends and strategies to enhance triacylglycerols and high-value compounds in microalgae. Algal Res. 2017;25:263-73.

43. Menon KR, Balan R, Suraishkumar GK. Stress induced lipid production in chlorella vulgaris: relationship with specific intracellular reactive species levels. Biotechnol Bioeng. 2013;110:1627-36.

44. Jakobsen AN, Aasen IM, Josefsen KD, Strøm AR. Accumulation of docosahexaenoic acid-rich lipid in Thraustochytrid aurantiochytrium sp. strain T66: effects of $\mathrm{N}$ and $\mathrm{P}$ starvation and $\mathrm{O}_{2}$ limitation. Appl Microbiol Biotechnol. 2008;80:297-306.

45. Lushchak VI. Adaptive response to oxidative stress: bacteria, fungi, plants and animals. Com Biochem Phys C. 2011;153:175-90.

46. Johansson M, Chen X, Milanova S, Santos C, Petranovic D. PUFA-induced cell death is mediated by Yca1p-dependent and -independent pathways, and is reduced by vitamin c in yeast. FEMS Yeast Res. 2016;16:fow007.

47. Ren LJ, Ji XJ, Huang H, Qu L, Feng Y, Tong QQ, Ouyang PK. Development of a stepwise aeration control strategy for efficient docosahexaenoic acid production by Schizochytrium sp. Appl Microbiol Biotechnol. 2010;87:1649-56.

48. Tocher DR. Metabolism and functions of lipids and fatty acids in teleost fish. Rev Fish Sci. 2003;11:107-84.

49. Liu B, Liu J, Sun P, Ma X, Jiang Y, Chen F. Sesamol enhances cell growth and the biosynthesis and accumulation of docosahexaenoic acid in the microalga Crypthecodinium cohnii. J Agric Food Chem. 2015;63:5640-5.

50. Gaffney M, O'Rourke R, Murphy R. Manipulation of fatty acid and antioxidant profiles of the microalgae Schizochytrium sp. through flaxseed oil supplementation. Algal Res. 2014;6:195-200

51. Chokshi K, Pancha I, Trivedi K, George B, Maurya R, Ghosh A, Mishra S. Biofuel potential of the newly isolated microalgae Acutodesmus dimorphus under temperature induced oxidative stress conditions. Bioresour Technol. 2015;180:162-71.

52. Yu Q, Liu Z, Xu H, Zhang B, Zhang M, Li M. Tio2 nanoparticles promote the production of unsaturated fatty acids (ufas) fighting against oxidative stress in Pichia pastoris. Rsc Adv. 2015;5:41033-40.

53. Kang NK, Lee B, Choi GG, Moon M, Min SP, Lim JK, Yang JW. Enhancing lipid productivity of Chlorella vulgaris using oxidative stress by tio 2 nanoparticles. Korean J Chem Eng. 2014;31:861-7.

54. Yilancioglu K, Cokol M, Pastirmaci I, Erman B, Cetiner S. Oxidative stress is a mediator for increased lipid accumulation in a newly isolated Dunaliella salina strain. PLOS ONE. 2014;9:e91957.

55. Tang D, Shi S, Li D, Hu C, Liu Y. Physiological and biochemical responses of Scytonema javanicum (cyanobacterium) to salt stress. J Arid Environ. 2007;71:312-20.

56. Kumar J, Singh VP, Prasad SM. NaCl-induced physiological and biochemical changes in two cyanobacteria Nostoc muscorum and Phormidium foveolarum acclimatized to different photosynthetically active radiation. J Photochem Photobiol B. 2015;151:221-32.

57. Cai $X$, Huang $Q$, Wang S. Isolation of a novel lutein-protein complex from Chlorella vulgaris and its functional properties. Food Funct. 2015;6:1893-9.

58. Ma Z, Tan Y, Cui G, Feng Y, Cui Q, Song X. Transcriptome and gene expression analysis of DHA producer Aurantiochytrium under low temperature conditions. Sci Rep. 2015;5:14446 
59. Cheng RL, Feng J, Zhang BX, Huang Y, Cheng J, Zhang CX. Transcriptome and gene expression analysis of an oleaginous diatom under different salinity conditions. Bioenerg Res. 2014;7:192-205.

60. Valledor L, Furuhashi T, Hanak AM, Weckwerth W. Systemic cold stress adaptation of chlamydomonas reinhardtii. Mol Cell Proteomics. 2013;12:2032-47.

61. Ren L, Hu X, Zhao X, Chen S, Wu Y, Li D, et al. Transcriptomic analysis of the regulation of lipid fraction migration and fatty acid biosynthesis in Schizochytrium sp. Sci Rep. 2017:7:3562.

62. Cheng YR, Sun ZJ, Cui GZ, Song X, Qiu C. A new strategy for strain improvement of Aurantiochytrium sp. based on heavy-ions mutagenesis and synergistic effects of cold stress and inhibitors of enoyl-acp reductase. Enzyme Microb Technol. 2016;93-94:182-90.
63. Qu L, Ji XJ, Ren LJ, Nie ZK, Feng Y, Wu WJ, et al. Enhancement of docosahexaenoic acid production by Schizochytrium sp. using a two-stage oxygen supply control strategy based on oxygen transfer coefficient. Lett Appl Microbiol. 2011;52:22-7.

64. Li X, Hu HY, Zhang YP. Growth and lipid accumulation properties of a freshwater Microalga scenedesmus sp. under different cultivation temperature. Bioresour Technol. 2011;102:3098-102.

65. Heath RL, Packer L. Photoperoxidation in isolated chloroplasts: I. kinetics and stoichiometry of fatty acid peroxidation. Arch Biochem Biophys. 1968;125:189-98.

\section{Submit your next manuscript to BioMed Central and we will help you at every step:}

- We accept pre-submission inquiries

- Our selector tool helps you to find the most relevant journal

- We provide round the clock customer support

- Convenient online submission

- Thorough peer review

- Inclusion in PubMed and all major indexing services

- Maximum visibility for your research

Submit your manuscript at www.biomedcentral com/submit 